\title{
O controle de autoridade no domínio bibliográfico: os catálogos digitais
}

\author{
Fabrício Silva Assumpção \\ Plácida Leopoldina Ventura Amorim da Costa Santos \\ Universidade Estadual Paulista "Júlio de Mesquita Filho" - UNESP, Brasil
}

Zaira Regina Zafalon

Universidade Federal de São Carlos - UFSCar, Brasil

REVIEW

\begin{abstract}
Resumo
Por meio de uma revisão de literatura, este estudo tem por objetivo analisar o controle de autoridade nos catálogos digitais. Para isso, inicia-se com uma breve introdução acerca do controle de autoridade, contextualiza a automação das bibliotecas e o surgimento dos catálogos digitais a partir da década de 1960 e destaca alguns pontos da história dos catálogos digitais, tais como a criação de formatos MAchine-Readable Cataloging (MARC) para dados bibliográficos e de autoridade. Como síntese da compreensão atual do controle de autoridade, apresenta os modelos conceituais Functional Requirements for Authority Data (FRAD) e Functional Requirements for Subject Authority Data (FRSAD). Por fim, descreve o modelo de dados utilizado nos catálogos digitais atuais e conclui ressaltando a presença do controle de autoridade na história da Catalogação, a importância dos modelos conceituais FRAD e FRSAD, a retomada dos registros de autoridade no código de catalogação Resource Description and Access (RDA), e a importância da compreensão do controle de autoridade no cenário atual da Catalogação.
\end{abstract}

Palavras-chave

Catalogação descritiva; Catálogos bibliográficos; Controle de autoridade; Functional Requirements for Authority Data (FRAD); Functional Requirements for Subject Authority Data (FRSAD); História da catalogação

\section{Authority control in the bibliographic domain: digital catalogs}

\begin{abstract}
In this paper we use a literature review to analyze authority control in digital catalogs. In order to do that, we start with a brief introduction to authority control, we contextualize library automation and the rising of digital catalogs from 1960s, and then we highlight some developments in the history of digital catalogs, such as the development of MAchine-Readable Cataloging (MARC) formats for bibliographic and authority data. After that, we present the Functional Requirements for Authority Data (FRAD) and the Functional Requirements for Subject Authority Data (FRSAD) as a synthesis of the current understanding about authority control. Finally, we describe the data model used in the current digital catalogs and we point out: the presence of authority control in the history of Cataloging, the value of FRAD and FRSAD conceptual models, the coverage of authority records by the Resource Description and Access (RDA), and the importance of the understanding about authority control in the current context of Cataloging.
\end{abstract}

Keywords

Authority control; Bibliographic catalogs; Descriptive cataloging; Functional Requirements for Authority Data (FRAD); Functional Requirements for Subject Authority Data (FRSAD); History of cataloging 


\section{Introdução}

No domínio bibliográfico, os catálogos destinam-se, de modo geral, à recuperação de informações sobre os recursos informacionais disponíveis no acervo de uma determinada instituição ou de um conjunto de instituições. Os recursos informacionais representados nos catálogos, por exemplo, livros, mapas, documentos audiovisuais, etc., estão relacionados a diversas entidades, tais como as pessoas e as entidades coletivas responsáveis por sua criação e os conceitos tratados em seu conteúdo.

Nos catálogos, os nomes pelos quais essas entidades são conhecidas são utilizados como base para os pontos de acesso que as representam. A falta de univocidade na relação entre as entidades e seus nomes fez surgir a necessidade do controle de autoridade, ou controle de vocabulário, de modo que a eficiência da recuperação da informação em um catálogo não fosse prejudicada pela omissão de registros bibliográficos relevantes e/ou pela recuperação de registros bibliográficos irrelevantes. Assim, o controle de autoridade é aqui entendido como um estado alcançado em um catálogo quando os pontos de acesso são utilizados de forma consistente, ou seja, quando: um ponto de acesso autorizado representa uma única entidade, uma entidade é representada por apenas um ponto de acesso autorizado e pontos de acesso não autorizados foram estabelecidos para guiar o usuário na utilização do catálogo. O controle de autoridade é alcançado mediante os procedimentos agrupados sob o termo trabalho de autoridade:

1. a criação de registros de autoridade (que, por sua vez, são utilizados para criar registros bibliográficos autorizados)

2. a compilação de registros em um arquivo de autoridade

3. a vinculação do arquivo de autoridade ao arquivo bibliográfico; juntos eles formam um sistema de autoridade

4. a manutenção do arquivo e do sistema de autoridade

5. a avaliação do arquivo e do sistema de autoridade (BURGER, 1985, p. 3, tradução nossa).

Este estudo é parte de uma pesquisa de doutorado que tem entre seus objetivos o entendimento das funções dos dados de autoridade nos catálogos. Para esse entendimento, são analisados os três principais tipos de catálogos observados ao longo da história da Catalogação: catálogos em livros, catálogos em fichas e catálogos digitais. Essa análise é conduzida em duas partes. A primeira parte, apresentada no artigo "O controle de autoridade no domínio bibliográfico: os catálogos em livros e em fichas", tem por objetivo analisar o controle de autoridade e o papel dos dados de autoridade nos catálogos em livros e em fichas. A segunda parte, apresentada neste artigo, tem por objetivo analisar o controle de autoridade nos catálogos digitais.

Este estudo, realizado a partir de uma revisão de literatura, justifica-se pela necessidade de compreensão do controle de autoridade nos catálogos, que pode auxiliar (1) no entendimento dos instrumentos de representação atualmente utilizados no domínio bibliográfico, (2) na construção de sistemas para catálogos digitais e (3) na avaliação e implementação de novos padrões voltados aos dados, registros e arquivos de autoridade.

Os resultados da segunda parte deste estudo são apresentados neste artigo em quatro seções: descrição e análise do controle de autoridade nos catálogos digitais (seção 2), breve apresentação dos modelos conceituais FRAD e FRSAD enquanto síntese da compreensão atual acerca dos dados de autoridade (seção 3), apontamentos acerca do modelo de dados dos catálogos digitais atuais (seção 4) e considerações finais (seção 5).

\section{0 controle de autoridade nos catálogos digitais}

A partir da década de 1960, algumas bibliotecas, principalmente nos Estados Unidos, começaram a utilizar computadores na tentativa de suprir as demandas decorrentes do cenário informacional no qual se encontravam: o rápido desenvolvimento econômico, científico e tecnológico e o aumento da quantidade de publicações científicas e tecnológicas com a vida útil cada vez menor (RAYWARD, 2002). Taylor (2006, p. 501, tradução nossa) contextualiza o início da automação das bibliotecas no cenário tecnológico da década de 1970: 
"Quando a automação de bibliotecas teve início na década de 1970, a maioria dos sistemas estava baseada em um modelo hierárquico envolvendo um computador mainframe e um ou mais terminais 'burros'”. Rayward (2002, p. 11, tradução nossa), ao traçar um histórico do uso de computadores em bibliotecas, sintetiza as vantagens inicialmente observadas que contribuíram para a automação das bibliotecas:

Os computadores pareciam oferecer aos bibliotecários a expectativa de um processamento mais eficiente, de serviços aprimorados aos usuários da biblioteca, de economia de dinheiro e contenção de custos, e de facilidade no compartilhamento de recursos e na cooperação entre bibliotecas, todas essas preocupações centrais ao tradicional funcionamento das bibliotecas.

A automação das bibliotecas no século XX pode ser dividida em quatro períodos: uso da computação offline (década de 1960 e início da década de 1970), surgimento dos sistemas online (década de 1970), surgimento dos microcomputadores e do CD-ROM (década de 1980) e revolução da Internet (década de 1990) (RAYWARD, 2002, p. 13). Com o surgimento dos sistemas online, no final da década de 1970 , os catálogos digitais passaram a ser denominados Online Public Access Catalogs (OPACs) (catálogos públicos de acesso online). Taylor e Joudrey (2009, p. 165) destacam que os catálogos online ocorreram com a convergência da capacidade de processamento e de armazenamento dos computadores, do baixo custo, dos softwares para a manipulação de arquivos extensos e dos próprios arquivos em um formato digital.

Os OPACs desenvolveram-se em diferentes localidades, em diferentes períodos e com diferentes intensidades de acordo com os recursos tecnológicos disponíveis. Ainda que com essas disparidades, os OPACs do século XX, de modo geral, podem ser entendidos por meio de duas gerações. A primeira geração, que surgiu no início da década 1980, possuía interface de menus e geralmente possibilitava a busca apenas por autores e títulos, sem pontos de acesso de assunto e remissivas (TAYLOR; JOUDREY, 2009, p. 167). No final de década de 1980, a segunda geração de OPACs já apresentava melhorias em relação à geração anterior:

Essa geração foi marcada pelas interfaces de usuário significativamente aprimoradas. Foi introduzida a busca com o uso de operadores booleanos, aumentando, assim, a quantidade de pontos de acesso disponíveis para busca. Isto significou que não era mais necessário que as buscas fossem realizadas com a palavra ou a frase exata ou com uma âncora; as palavras agora poderiam corresponder à busca, mesmo se estivessem no meio de uma cadeia de caracteres. O processo de busca também foi melhorado com o uso de truncamento e de caracteres curinga, a capacidade de navegação (incluindo a navegação pelo índice de termos), o uso de todo o registro MARC, os refinamentos de busca interativos e o acesso por assunto. A segunda geração de OPACs trouxe também uma maior manipulação dos resultados de busca e melhores sistemas de ajuda com mensagens de erro mais informativas [...]. (TAYLOR; JOUDREY, 2009, p. 167-168, tradução nossa).

Sobre o uso de computadores especificamente nos processos de catalogação, McCallum (2002, p. 35) aponta que a ideia de obter eficiência nos fluxos de trabalho, principalmente pelo rápido compartilhamento de registros, foi a principal força que direcionou os experimentos para automação das bibliotecas. Mesmo com essas vantagens, os processos de catalogação, entre os processos realizados nas bibliotecas, não foram os primeiros a serem automatizados, cabendo essa posição aos processos de circulação e de inventário das coleções (MCCALLUM, 2002, p. 34). A autora explica que os processos de catalogação e de manutenção dos catálogos apresentavam complexos requisitos, o que fez com que eles não começassem a ser automatizados antes da metade da década de 1960, quando a Library of Congress (LC) iniciou os estudos para o MARC (MAchineReadable Cataloging) Pilot Project (AVRAM, 1968; MCCALLUM 2002, p. 34).

Como resultado de seus estudos, a LC deu início em 1966 a um serviço de distribuição de registros bibliográficos em fitas magnéticas, possibilitado pelo uso do Formato MARC (AVRAM, 1968). Esse formato consistia em especificações e designadores de conteúdo que, aplicados a registros bibliográficos, possibilitava a identificação e o processamento de seus dados por programas de computador, e, junto de uma estrutura genérica posteriormente reconhecida como as normas ANSI/NISO Z39.2 e ISO 2709, permitia a gravação desses registros bibliográficos em fitas magnéticas. Inicialmente, esse formato foi utilizado para a impressão de fichas catalográficas, de catálogos em livros e em microfichas e, posteriormente, para a criação de catálogos locais online (MCCALLUM, 2002, p. 43; TAYLOR, 2006, p. 498).

A partir da experiência com o Formato MARC, a LC lançou em 1969 o Formato MARC II e, na década seguinte, já eram encontradas diversas variações do formato original em vários países. 
Para Clack (1988, p. 37), problemas no controle de autoridade são mais evidentes nos catálogos digitais que nos catálogos manuais. Segundo a autora, quando os computadores começaram a ser utilizados nas bibliotecas, houve o falso entendimento de que todos os problemas relacionados ao acesso aos registros bibliográficos seriam automaticamente solucionados e que a integridade desses registros não seria mais necessária, o que levou muitas bibliotecas a converterem seus catálogos manuais em digitais e rapidamente constatarem que seus catálogos digitais eram falhos em prover acesso completo e fácil aos registros bibliográficos e que o controle de autoridade era necessário em alguma medida para melhorar o acesso (CLACK, 1988, p. 37).

Assim como nos catálogos em livros e em fichas, nos catálogos digitais os registros bibliográficos estão sujeitos a alterações em decorrência de diversos fatores. Os pontos de acesso podem ser alterados, por exemplo, devido às mudanças nas regras de catalogação, à descoberta de novos dados sobre as entidades (datas de nascimento e morte, sobrenomes omissos, etc. de uma pessoa, por exemplo) ou à substituição de um termo por outro mais apropriado para representar um determinado conceito. Para Malinconico (1975, p. 215, tradução nossa), a manutenção dos catálogos digitais para a realização de alterações como essas requer a atenção a dois problemas básicos: "(1) a necessidade de garantir a consistência a qualquer momento, e (2) a necessidade de prover um método para a alteração rápida e precisa dessa estrutura de acordo com as circunstâncias". Partindo dessas necessidades, o autor descreve o arquivo de autoridade digital implantado na New York Public Library em 1970 para auxiliar na criação, na gestão e no uso do catálogo dessa biblioteca, sendo esse, talvez, um dos primeiros arquivos de autoridade digitais relatados na literatura.

Partindo da necessidade do controle de autoridade em catálogos digitais e com o intuito de promover a automação dos arquivos de autoridade, em 1976, a LC publicou a edição preliminar do documento Authorities: a MARC Format: Specifications for Magnetic Tapes Containing Authority Records, provendo especificações e designadores de conteúdo para registros de autoridade de nomes, de títulos uniformes e de assuntos, de modo que pudessem ser gravados em fitas magnéticas utilizando a mesma estrutura genérica utilizada com os registros bibliográficos (MARC DEVELOPMENT OFFICE, 1976; BURGER, 1985, p. 74).

A edição preliminar do formato foi utilizada para a distribuição de registros de autoridade nos Estados Unidos e na Europa até 1981, quando a LC lançou a primeira edição do Authorities: a MARC Format, que continha adendos à edição preliminar do formato, tais como a cobertura de pontos de acesso relativos a séries (BURGER, 1985, p. 74). Desde então, a LC tem mantido e atualizado esse formato, hoje denominado MARC 21 Format for Authority Data (Formato MARC 21 para Dados de Autoridade) e integrante do conjunto de Formatos MARC 21.2 Acompanhando os desenvolvimentos realizados nos Estados Unidos em relação à automação dos arquivos de autoridade, outros formatos para dados de autoridade surgiram, tais como o UNIMARC/Authorities: Universal Format for Authorities, cuja primeira edição é de 1991.

Convém ressaltar que nem sempre o controle de autoridade esteve presente nos catálogos digitais e que, ainda hoje, existem catálogos que, por motivos diversos e alheios aos objetivos desta pesquisa, carecem de qualquer atividade de controle dos pontos de acesso. Sobre a inclusão do controle de autoridade nos catálogos digitais, Brandt, no final da década de 1980, escreveu que
À medida que mais e mais bibliotecas migram para catálogos online, o controle de autoridade está sendo visto de perto como uma parte do gerenciamento de dados para assegurar a consistência e a integridade das entradas, assim como para relacionar registros que compartilham um cabeçalho. Quando aplicado às bases de dados bibliográficos, o controle de autoridade é mais que um meio usado para estabelecer e relacionar os cabeçalhos - ele pode automaticamente e literalmente "vincular" os registros. Como tal, o controle de autoridade pode ser visto como uma ferramenta essencial para a administração de dados. (BRANDT, 1988, p. 297, tradução nossa).

O crescente uso de catálogos e de arquivos de autoridade digitais, com o surgimento de redes e de programas para o compartilhamento de registros bibliográficos e de autoridade, impulsionou no domínio bibliográfico os esforços para a criação de padrões, diretrizes e modelos que favorecessem o compartilhamento de dados catalográficos em âmbito internacional. Alguns desses esforços proveem um entendimento atual e sistematizado, ainda que não seja consensual, acerca dos dados de autoridade, como é caso dos modelos conceituais FRAD e FRSAD, apresentados na seção seguinte. 


\section{Os modelos conceituais FRAD e FRSAD}

A automação dos arquivos de autoridade trouxe diversas vantagens para as bibliotecas, como destaca Maxwell (2002, p. 11, tradução nossa):

O armazenamento de dados de autoridade em forma legível por máquina apresenta vantagens óbvias em relação aos arquivos em fichas, incluindo a facilidade de manipulação dos dados, a flexibilidade na apresentação e as capacidades de busca melhoradas, mas também abre a possibilidade de compartilhamento dos dados de autoridade entre bibliotecas, diminuindo, assim, o custo dessa importante tarefa.

O compartilhamento de dados, em qualquer domínio, requer padronização. A capacidade de compartilhamento dos dados resultantes dos processos de catalogação, compreendida como uma importante vantagem da automação de bibliotecas, pressupõe a existência de padronização entre as instituições envolvidas neste compartilhamento.

No domínio bibliográfico, o anseio pelo compartilhamento de dados pode ser observado já em períodos remotos como na Idade Média, quando, no século XIII, monges ingleses elaboraram um catálogo coletivo representando obras presentes em 183 bibliotecas monásticas (SANTOS; PEREIRA, 2014, p. 17). De forma mais intensa, o anseio por esse compartilhamento pode ser notado a partir da segunda metade do século XIX com duas principais ações ainda voltadas aos catálogos analógicos: o desenvolvimento de códigos de catalogação de amplo alcance e a consolidação do uso de fichas catalográficas em um tamanho padrão (7,5 x 12,5 cm). Com a introdução do uso de computadores nas atividades de catalogação, a partir da segunda metade do século XX, o anseio pelo compartilhamento de dados levou ao desenvolvimento de meios para a padronização e a troca de informações já em ambientes digitais, entre esses meios, o Formato MARC e os formatos dele derivados, as normas ANSI/NISO Z39.2 e ISO 2709 e o protocolo Z39.50.

Como parte dos seus esforços para o controle bibliográfico universal, a International Federation of Library Associations and Institutions (IFLA) tem publicado padrões, orientações e formatos no intuito de, a partir da padronização, favorecer o compartilhamento de dados catalográficos em uma escala global. Algumas dessas publicações destinam-se aos dados de autoridade, entre elas: Guidelines for authority and reference entries (1984), com uma segunda edição publicada em 2001 sob o título Guidelines for authority records and references; Management and use of name authority files: personal names, corporate bodies and uniform titles: evaluation and prospects (1991); UNIMARC/Authorities: Universal Format for Authorities (1991), com segunda e terceira edições publicadas em 2001 e 2009, respectivamente; Mandatory data elements for internationally shared resource authority records (1998).

Ao final do século $\mathrm{XX}$, o domínio bibliográfico, com seus instrumentos de representação estabelecidos principalmente a partir de uma realidade existente décadas antes, passou a enfrentar um novo cenário:

As bibliotecas na década de 1990 foram forçadas a começar a reconsiderar seus padrões e práticas relacionados à organização da informação bibliográfica, por um lado, considerando na prática cotidiana a maturidade do pensamento teórico e os padrões desenvolvidos internacionalmente, e, por outro, a pressão para incluir a descrição de novos formatos de documentos - recursos eletrônicos (nascidos digitais ou digitalizados) e, posteriormente, documentos da Web - em seus catálogos de modo a satisfazer as necessidades dos usuários. (WILLER; DUNSIRE, 2013, p. 2, tradução nossa).

Com isso, um processo de questionamento dos conceitos e das teorias tradicionais foi iniciado em 1990 com um seminário sobre registros bibliográficos realizado pela IFLA em Estocolmo, na Suécia (WILLER; DUNSIRE, 2013, p. 13). Willer e Dunsire (2013, p. 13, tradução nossa) explicam que

Foi um momento em que a necessidade de mudança estava no ar e impulsionada por muitos fatores, incluindo o desenvolvimento e o crescimento de sistemas automatizados e de bases de dados nacionais e internacionais de larga escala, a necessidade de reduzir os custos com a catalogação por meio do reuso dos registros bibliográficos existentes, o crescimento contínuo das publicações, especialmente das publicações eletrônicas, e uma ampliação da variedade de expectativas e de necessidades dos usuários.

e que os participantes do seminário

[...] admitiram que "o ambiente em que os princípios e padrões da catalogação operavam havia mudado dramaticamente" e "reconheceram a importância de satisfazer as necessidades 
do usuário e abordar mais efetivamente a ampla variedade de necessidades associadas aos vários tipos de materiais e aos vários contextos em que os registros bibliográficos são utilizados". (WILLER; DUNSIRE, 2013, p. 13, tradução nossa).

Assim, com o intuito de relacionar os dados dos registros bibliográficos às necessidades dos usuários e de recomendar um nível básico de funcionalidade para os registros criados por agências bibliográficas nacionais (IFLA STUDY GROUP ON THE FRBR, 1998, p. 7), um grupo da IFLA conduziu um estudo que resultou no modelo conceitual Functional Requirements for Bibliographic Records (Requisitos Funcionais para Registros Bibliográficos), mais conhecido como FRBR, publicado em 1998. Esse modelo apresenta um conjunto de entidades de interesse do universo bibliográfico, os atributos que caracterizam cada uma dessas entidades e os relacionamentos existentes entre elas, assim como ações, denominadas tarefas dos usuários, que demandam tais atributos e relacionamentos.

Desde sua publicação, o FRBR tem influenciado de forma significativa o desenvolvimento da Catalogação. Segundo Coyle (2016, p. 65, tradução nossa),

O modelo de dados bibliográficos FRBR é a mudança de pensamento mais radical sobre os catálogos bibliográficos desde que Panizzi desenvolveu suas noventa e uma regras para o catálogo do British Museum. [...] O FRBR é alternadamente visto como uma análise das necessidades do usuário, como uma descrição do fluxo de catalogação e como um modelo de dados para um futuro formato de registro bibliográfico. No entanto, ele é raramente visto como originalmente foi projetado para ser: um desenvolvimento dos requisitos básicos para um padrão internacional de registros bibliográficos nacionais.

Entre as recomendações do grupo de estudo responsável pelo FRBR estava a necessidade de expansão do modelo para a cobertura dos dados de autoridade (IFLA STUDY GROUP ON THE FRBR, 1998, p. 5). Partindo dessa recomendação, em 1999 a IFLA iniciou o Working Group on Functional Requirements and Numbering of Authority Records (FRANAR) (Grupo de trabalho sobre requisitos funcionais e numeração de registros de autoridade), voltado aos registros de autoridade (IFLA WORKING GROUP ON FRANAR, 2013, p. i). O resultado do estudo realizado pelo FRANAR foi publicado em 2009 sob o título Functional Requirements for Authority Data: a conceptual model (Requisitos Funcionais para Dados de Autoridade: um modelo conceitual), passando a ser conhecido pela sigla FRAD (IFLA WORKING GROUP ON FRANAR, 2013).

O FRAD, cujo escopo limita-se aos dados de autoridade referentes a nomes e títulos, excluindo, portanto, os dados de autoridade referentes aos conceitos ("assuntos"), tem como propósito principal

[...] prover um quadro para a análise dos requisitos funcionais para o tipo de dado de autoridade requerido para apoiar o controle de autoridade e para o compartilhamento internacional de dados de autoridade. O modelo foca nos dados, independentemente de como eles são acondicionados (e.g., em registros de autoridade). (IFLA WORKING GROUP ON FRANAR, 2013, p. 1, tradução nossa).

Utilizando-se da técnica de análise de entidades, atributos e relacionamentos e partindo do entendimento de que as entidades bibliográficas são conhecidas por nomes e/ou identificadores e que esses nomes e identificadores são utilizados como base para os pontos de acesso controlados, são definidas no FRAD dezesseis entidades (pessoa, família, entidade coletiva, obra, expressão, manifestação, item, conceito, objeto, evento, lugar, nome, identificador, ponto de acesso controlado, regras e agência) com seus atributos e relacionamentos (IFLA WORKING GROUP ON FRANAR, 2013).

Assim como no FRBR, no FRAD são definidas tarefas dos usuários. Neste modelo, são compreendidos como usuários aqueles que criam os dados de autoridade e aqueles que utilizam os dados de autoridade, direta ou indiretamente, por meio dos pontos de acesso controlados presentes nos catálogos, nas bibliografias nacionais e em outras bases de dados similares (IFLA WORKING GROUP ON FRANAR, 2013, p. 46). As quatro tarefas dos usuários definidas no FRAD são:

- encontrar: encontrar uma entidade ou um conjunto de entidades correspondente ao critério estipulado (ou seja, encontrar uma única entidade ou um conjunto de entidades utilizando como critério de busca um atributo, a combinação de atributos ou um relacionamento da entidade); ou explorar o universo das entidades bibliográficas utilizando seus atributos e relacionamentos;

- identificar: identificar uma entidade (ou seja, confirmar se a entidade representada corresponde à entidade buscada, distinguir entre duas ou mais entidades com 
características similares) ou validar a forma do nome para ser utilizada como um ponto de acesso controlado;

- contextualizar: localizar uma pessoa, entidade coletiva, obra, etc. no contexto; esclarecer o relacionamento entre duas ou mais pessoas, entidades coletivas, obras, etc.; ou esclarecer o relacionamento entre uma pessoa, entidade coletiva, etc. e o nome pelo qual essa pessoa, entidade coletiva, etc. é conhecida (por exemplo, o nome utilizado na religião versus o nome secular);

- justificar: documentar a razão pela qual o criador dos dados de autoridade escolheu o nome ou a forma do nome na qual o ponto de acesso controlado está baseado (IFLA WORKING GROUP ON FRANAR, 2013, p. 46, tradução nossa).

Enquanto o FRANAR trabalhava no desenvolvimento do FRAD, outro grupo de trabalho foi estabelecido pela IFLA, dessa vez com o foco nos dados de autoridade de assunto. O Working Group on the Functional Requirements for Subject Authority Records (FRSAR) (Grupo de trabalho sobre os requisitos funcionais para registros de autoridade de assunto), formado em 2005, atuou até 2010, quando foi publicado seu relatório final intitulado Functional Requirements for Subject Authority Data (FRSAD) (Requisitos funcionais para dados de autoridade de assunto).

Neste relatório, o grupo de trabalho apresenta um modelo construído a partir do FRBR e utilizando também a técnica de análise de entidades, atributos e relacionamentos. O FRSAD define as entidades thema e nomen, que se relacionam à entidade obra, definida no FRBR: uma obra tem como assunto um ou mais themas; um thema é assunto de uma ou mais obras; um thema tem como denominação um ou mais nomens; e um nomen é a denominação de um ou mais themas (IFLA WORKING GROUP ON FRASAR, 2010).

Como potenciais usuários dos dados de autoridade de assunto, o FRSAD define: (a) profissionais da informação que criam e mantêm dados de autoridade de assunto, incluindo catalogadores e criadores de vocabulários controlados; (b) profissionais da informação que criam e mantêm metadados; (c) bibliotecários de referência e outros profissionais da informação que buscam informações como intermediários; e (d) usuários finais que buscam informações para satisfazer suas necessidades informacionais (IFLA WORKING GROUP ON FRASAR, 2010, p. 33, tradução nossa). As tarefas realizadas por esses usuários são:

- encontrar um ou mais assuntos e/ou suas denominações que correspondam ao critério de busca estipulado, utilizando atributos e relacionamentos;

- identificar um assunto e/ou sua denominação baseado em seus atributos ou relacionamentos (ou seja, distinguir entre dois ou mais assuntos ou denominações com características similares e confirmar que o assunto ou a denominação apropriada foi encontrada);

- selecionar um assunto e/ou uma denominação apropriada às necessidades do usuário (ou seja, escolher ou rejeitar com base nos requisitos e nas necessidades do usuário);

- explorar os relacionamentos entre assuntos e/ou suas denominações (ou seja, explorar os relacionamentos para entender a estrutura de um domínio de assunto e sua terminologia) (IFLA WORKING GROUP ON FRASAR, 2010, p. 33, tradução nossa).

Os três modelos conceituais desenvolvidos pela IFLA (FRBR, FRAD e FRSAD), por terem diferentes objetivos, abordam um mesmo cenário - o universo bibliográfico - a partir de pontos de vistas distintos, o que faz com eles apresentem entidades, atributos, relacionamentos e tarefas dos usuários que, em alguns casos, sobrepõem-se ou opõem-se. Atualmente, as divergências e as similaridades entre esses modelos estão em discussão na proposta de um FRBR - Library Reference Model (FRBR-LRM) (FRBR REVIEW GROUP, 2016).

\section{0 modelo de dados dos catálogos digitais}

Ao olhar para os catálogos digitais, para a função exercida pelos dados de autoridade e para os modelos conceituais FRAD e FRSAD é necessário considerar que, mesmo que os catálogos digitais tenham apresentado considerável desenvolvimento nas últimas décadas, dispondo de novas possibilidades de busca, de navegação 
e de interface, o modo com que os dados bibliográficos e de autoridade estão dispostos e relacionados sofreu pouca ou nenhuma alteração, visto que o modelo de dados, implantado com o Formato MARC II ainda na década de 1970 e posteriormente complementado com o formato para dados de autoridade, é utilizado ainda hoje na construção de catálogos digitais.

Neste estudo, um modelo é considerado "[...] uma descrição simplificada de certos aspectos da realidade utilizada para compreender, estruturar ou predizer partes do mundo real" (HITZLER; KRÖTZSCH; RUDOLPH, 2010, p. 2, tradução nossa). Para Coyle (2016, p. 29, tradução nossa)

Há várias razões para se criar modelos do mundo real, a maior parte delas tem a ver com a dificuldade de manipular o mundo real diretamente. Arquitetos criam modelos de prédios que desenharam, projetistas criam modelos de argila de novos projetos automobilísticos e químicos criam modelos físicos para representar moléculas. Muitas vezes, nosso modelo do mundo não é um modelo físico, mas um modelo de dados simbólico. Esses modelos são abstrações do mundo real e sua semelhança com a realidade é mais conceitual do que física.

Sayão (2001, p. 86), em um estudo sobre os modelos teóricos na Ciência da Informação, destaca a proposta de Burt e Kinnucan (1990) acerca de uma configuração contínua em que se situam os tipos de modelos de informação. De acordo com essa proposta, em um extremo do contínuo situa-se o ser humano com seu conhecimento, enquanto que no outro extremo está o sistema de informação e, entre os dois, o campo de representações: "[...] o espaço onde se encontram representações de uma ou de outra realidade. Essas representações experimentam criar pontes ou elucidar algumas ou todas as estranhezas que se supõe existam entre essas realidades" (SAYÃO, 2001, p. 86).

Os modelos que estão mais próximos do que seja a representação do usuário humano e do que se passa em sua cabeça em relação ao sistema são denominados modelos cognitivos; ao passo que os modelos que se identificam com o sistema e tentam descrever o que se passa em seu interior são mais conhecidos como modelos de dados. Na região intermediária do contínuo, é o lugar geométrico dos modelos que interpretam os usuários, o sistema e a interação entre eles; os modelos que se enquadram nesta categoria são coletivamente denominados de modelos conceituais. (SAYÃO, 2001, p. 86).

No âmbito dos bancos de dados, Fusco (2011, p. 63) considera um modelo de dados "[...] uma representação utilizada para descrever a estrutura conceitual, lógica e física de um banco de dados, esta estrutura compreende objetos de dados, características desses dados, relacionamentos e regras que restringem esses dados num cenário." Para o autor, os modelos de dados podem ser construídos em três níveis:

- Modelo de dados conceitual: fornece uma visão abstrata que representa os dados no domínio do cenário;

- Modelo de dados lógico: fornece uma visão numa representação de uma estrutura de modelo de banco de dados;

- Modelo de dados físico: fornece uma visão mais detalhada de como os dados estão armazenados no banco de dados. (FUSCO, 2011, p. 63)

Partindo desse entendimento, o modelo de dados dos catálogos digitais abordado neste estudo é um modelo de dados conceitual, uma vez que não se refere à estrutura do banco de dados em si (modelo de dados lógico) e nem ao armazenamento no banco de dados (modelo de dados físico), mas sim a uma visão abstrata acerca do domínio (o catálogo). Esse modelo de dados dos catálogos digitais, representado na Figura 1, pode ser descrito da seguinte forma:

- cada edição ou versão de um recurso informacional é representada por um registro bibliográfico;

- cada registro bibliográfico contém a descrição bibliográfica da edição ou versão do recurso informacional, os pontos de acesso estabelecidos para sua localização no catálogo e os dados administrativos sobre o recurso informacional e/ou sobre o registro;

- a cada registro bibliográfico podem ser vinculados registros de itens para indicar os exemplares daquela edição ou versão do recurso informacional que fazem parte da coleção de uma determinada instituição; 
- a cada registro bibliográfico podem ser vinculados outros registros bibliográficos para indicar recursos informacionais relacionados;

- $\quad$ registros de autoridade podem ser criados para controlar os pontos de acesso utilizados nos registros bibliográficos;

- nos registros de autoridade, além do ponto de acesso autorizado, podem ser incluídos pontos de acesso variantes, pontos de acesso relacionados e dados que apoiem o uso e a gestão do registro de autoridade;

- um ponto de acesso relacionado é um ponto de acesso autorizado controlado por outro registro de autoridade;

- o uso de pontos de acesso relacionados cria vínculos entre registros de autoridade;

- o uso de um ponto de acesso autorizado em um registro bibliográfico cria um vínculo, implícito ou explícito, entre o registro bibliográfico e o registro de autoridade que controla o ponto de acesso.

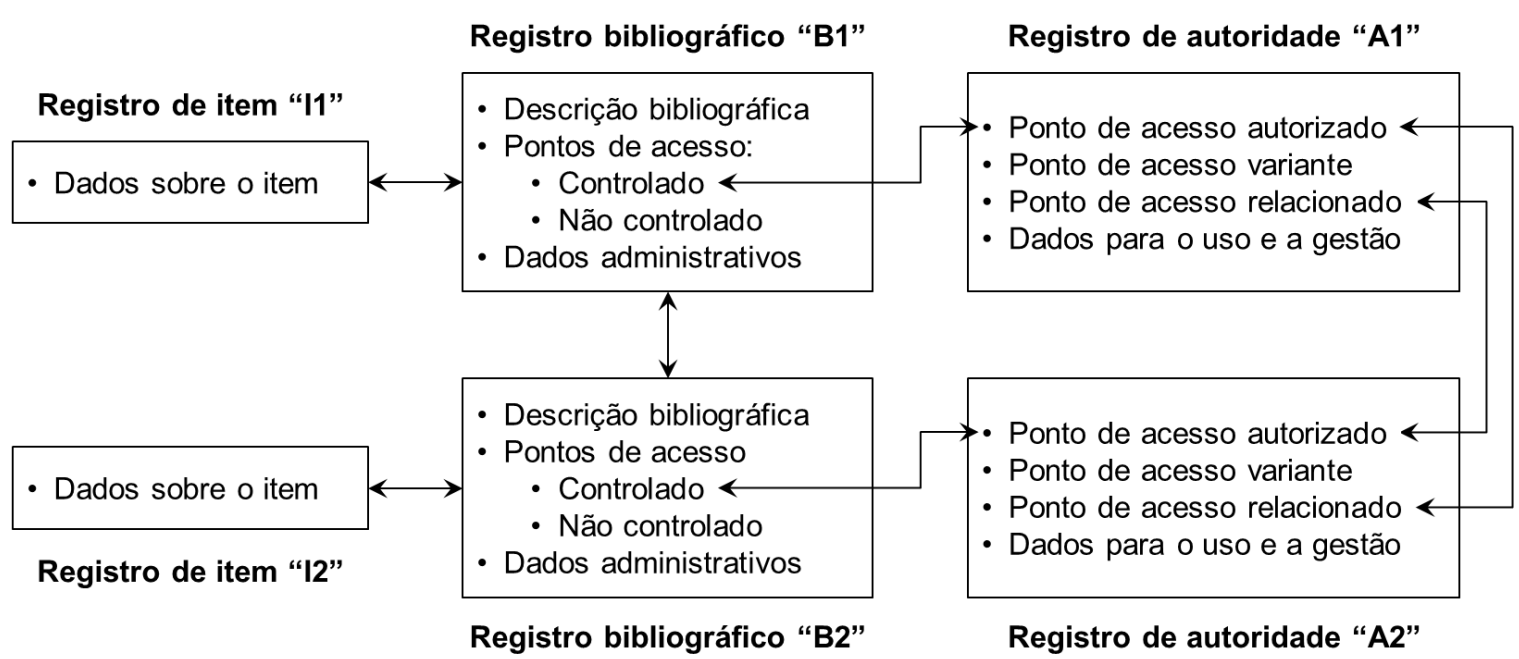

Figura 1 - Modelo de dados dos catálogos digitais

Fonte: Elaborada pelo autor.

Os tipos de vínculos entre os registros bibliográficos e de autoridade decorrem do tipo de relacionamento entre o catálogo e o arquivo de autoridade. Para os propósitos desta pesquisa, considera-se que nos catálogos digitais são notados dois tipos de vínculos: implícitos e explícitos. Esses vínculos decorrem de dois tipos de relacionamentos entre arquivo de autoridade e catálogo listados por Burger (1985, p. 32-34):

- vínculos implícitos ocorrem quando o arquivo de autoridade é independente do catálogo, quando os dois existem como bases de dados distintas, sem que as alterações realizadas no arquivo de autoridade sejam automaticamente refletidas no catálogo; vínculos implícitos são observados, por exemplo, nos casos em que os pontos de acesso autorizados utilizados em um catálogo são obtidos a partir de um arquivo de autoridade externo à instituição mantenedora do catálogo, sem que haja, localmente, um arquivo de autoridade; instituições que não possuem um arquivo de autoridade e utilizam o arquivo de autoridade da Biblioteca Nacional, por exemplo, enquadram-se nessa categoria;

- vínculos explícitos ocorrem quando o arquivo de autoridade está conectado ao catálogo, quando os dois estão vinculados de modo que uma alteração realizada no arquivo de autoridade é automaticamente refletida no catálogo; exemplos de vínculos explícitos são notados nos sistemas de gerenciamento de bibliotecas que permitem a criação de registros de autoridade e sua vinculação aos registros bibliográficos. 
Além de influenciarem a gestão do catálogo, permitindo, por exemplo, a realização de alterações de forma automática, os tipos de vínculos entre registros bibliográficos e registros de autoridade podem influenciar também a consulta ao catálogo. Em relação a isso, são descritos na literatura dois cenários: (1) ao buscar por um ponto de acesso não autorizado, o usuário é orientado a realizar a busca utilizando o ponto de acesso autorizado; e (2) ao buscar por um ponto de acesso não autorizado, o usuário é automaticamente direcionado para os resultados de uma busca utilizando o ponto de acesso autorizado (BURGER, 1985, p. 43).

Enquanto nos catálogos em livros e em fichas o acesso aos registros bibliográficos dava-se somente pelos pontos de acesso localizados no início das entradas, nos catálogos digitais qualquer dado presente em um registro, e não somente os nomes dos responsáveis, os títulos e os termos escolhidos, pode ser considerado um ponto de acesso (CARPENTER, 1989, p. 79; TAYLOR; JOUDREY, 2009, p. 39).

O acesso aos registros em um catálogo online é limitado somente ao que não está descrito. Qualquer elemento de metadado (tal como o publicador ou o ilustrador) utilizado em uma descrição bibliográfica pode servir como um ponto de acesso e a busca pode incorporar uma variedade de sofisticados movimentos não lineares. (SVENONIUS, 2000, p. 23, tradução nossa)

Complementando a descrição dos catálogos digitais, Taylor (2006, p. 12, tradução nossa) explica que eles não possuem entradas no mesmo sentido que os catálogos em livros ou em fichas, em que a descrição de um recurso era replicada, em sua totalidade ou não, a cada entrada necessária, uma vez que nos catálogos online

Uma cópia de cada registro bibliográfico é armazenada pelo sistema. Índices são gerados para vincular cada nome, título, cabeçalho de assunto ou outra entidade utilizada como ponto de acesso aos registros bibliográficos associados. Em resposta a uma busca, os elementos de dados selecionados de cada registro bibliográfico relevante são copiados para a tela do usuário em um formato de apresentação escolhido para aquele sistema.

O arranjo dos registros bibliográficos nos catálogos em livros permitia que as obras de um mesmo responsável, com suas diferentes edições ou versões, fossem apresentadas como um conjunto ao usuário, provendo a este uma visão geral das obras. O arranjo dos registros bibliográficos nos catálogos em fichas, em que as diferentes edições de uma obra eram registradas em fichas individuais apresentadas segundo uma ordem alfabética específica, reduziu a capacidade do catálogo de, com o máximo possível de economia, prover uma visão geral de todas as obras, edições e versões relacionadas a um responsável. Sobre o arranjo dos registros bibliográficos em catálogos digitais, Chan (2007, p. 25, tradução nossa) aponta que

O modo com que os registros são arranjados internamente em um catálogo é uma questão de design do sistema e afeta os usuários finais somente no sentido de que um sistema pode ser mais fácil de utilizar e aparentemente recuperar resultados mais satisfatórios que o outro. Mas, como os itens recuperados são apresentados na tela também depende do design do sistema e, aqui, os usuários finais devem estar cientes de que mais opções estão abertas do que aquelas disponíveis no uso de um catálogo manual. Os resultados de uma busca são normalmente apresentados em uma ordem padrão, por data ou por nome, mas, na maioria dos sistemas, a lista pode ser reordenada de acordo com as preferências dos usuários.

Assim como ocorreu com os catálogos digitais, melhorias foram implementadas com a evolução dos arquivos de autoridade digitais no decorrer dos anos. O estabelecimento de vínculos automáticos entre registros bibliográficos e de autoridade, descrita por Burger (1985, p. 32-34) e por Brandt (1988, p. 297), é um exemplo dessas melhorias.

A publicação do FRBR no final da década de 1990 deu origem a uma série de estudos teóricos e aplicações práticas na tentativa da utilização desse modelo conceitual como modelo de dados para os catálogos digitais. Essas tentativas, de modo geral, mostraram-se infrutíferas e permaneceram em carácter experimental, de modo que, por motivos alheios aos objetivos deste estudo, o modelo conceitual FRBR não se consolidou enquanto principal referencial para o desenvolvimento de catálogos.

\section{Considerações finais}

Partindo do objetivo de analisar o controle de autoridade nos catálogos digitais foram tratados neste artigo, por meio de uma revisão de literatura, alguns marcos do desenvolvimento dos catálogos digitais e os modelos conceituais FRAD e FRSAD, assim como considerações sobre o modelo de dados dos catálogos digitais atuais. 
Como destacado na introdução, este artigo e o artigo "O controle de autoridade no domínio bibliográfico: os catálogos em livros e em fichas" são parte de uma pesquisa que tem entre seus objetivos o entendimento das funções dos dados de autoridade nos catálogos em livros, em fichas e digitais. Com esses dois artigos, nota-se que a presença dos dados de autoridade pode ser observada ao longo dos últimos 150 anos da história da Catalogação, evoluindo desde o uso de remissivas nos catálogos em livros, passando pelas fichas de autoridade e chegando até os sistemas de autoridade em ambientes digitais, que têm potencial para o compartilhamento de dados em uma escala global.

Ao longo de sua evolução, os dados de autoridade acumularam funções como a de permitir aos usuários, sejam eles seus criadores ou seus utilizadores, encontrar, identificar, selecionar, contextualizar, justificar e explorar o universo bibliográfico, como definido nos modelos conceituais FRAD e FRSAD. Além dessa função, os dados de autoridade, em especial os pontos de acesso, atuam como dispositivos de ligação ("linking devices"), conectando os registros bibliográficos de recursos informacionais relacionados (TILLETT, 1992, p. 23).

Sobre a importância dos modelos conceituais FRAD e FRSAD, entende-se aqui que esses modelos, por definirem entidades, atributos, relacionamentos e tarefas dos usuários, apresentam uma sistematização do entendimento atual acerca do controle de autoridade nos catálogos. Essa sistematização, construída a partir de uma metodologia consolidada (a análise das entidades, atributos e relacionamentos) é de fundamental importância em tempos de mudanças como o vivenciado atualmente no domínio bibliográfico. Por meio dessa sistematização, assim como daquela apresentada no modelo FRBR, explicita-se que o domínio bibliográfico, com suas ferramentas de acesso à informação e seus instrumentos e processos de representação, fundamentase em teorias e não somente na atividade prática.

Outro ponto de destaque é a retomada dos registros de autoridade por parte de um código de catalogação anglo-americano. A segunda edição preliminar do código de catalogação da American Library Association (ALA), publicado em 1941, continha, em um apêndice, instruções para a criação de registros de autoridade. Essas instruções foram removidas da segunda edição deste código e do AACR, que o sucedeu. Como sucessor do AACR tem-se o Resource Description and Access (RDA), publicado em 2010 e em processo de implantação em alguns países. O RDA, que tem entre seus fundamentos o FRAD, traz instruções sobre elementos de dados voltados aos registros de autoridade, favorecendo, assim, a criação desses registros (ASSUMPÇÃO; SANTOS, 2013).

A história da Catalogação é permeada pelo uso de tecnologias: técnicas de impressão, fichas catalográficas e formatos para o intercâmbio de dados são algumas delas, sendo que a revolução tecnológica decorrente do uso de computadores trouxe novos meios para o alcance dos objetivos da organização da informação e forçou uma reconsideração sobre a forma e a função dos registros bibliográficos (SVENONIUS, 2000, p. 107) e, consequentemente, dos registros de autoridade.

O surgimento da Web no início da década de 1990, sem dúvida, significou uma revolução no modo de compartilhar informações. Os catálogos digitais, com seus dados bibliográficos e de autoridade, passaram a ser disponibilizados na Web e, apesar do novo ambiente tecnológico, os instrumentos de descrição, regras de catalogação e formatos para o intercâmbio de dados, principalmente, mantiveram-se em certa medida imutáveis, contribuindo, assim, para uma reprodução na Web do mesmo modelo de dados utilizado nos catálogos digitais anteriores a ela.

Mais recentemente, começaram a surgir no contexto brasileiro as discussões sobre a disponibilização de dados bibliográficos e de autoridade utilizando as tecnologias da Web Semântica e as práticas de Linked Data, de modo que os catálogos possam se beneficiar do relacionamento e do reuso de dados e possam beneficiar, com seus dados, outros espaços e serviços de informação.

Frente aos desafios impostos por esse cenário, a compreensão do controle de autoridade é essencial. Essa compreensão deve estender-se desde os princípios que norteiam o controle de autoridade, incluindo os objetivos dos catálogos, revitalizados como as tarefas dos usuários, que dependem do controle de autoridade, até a identificação daquelas práticas que são hoje reproduzidas, mas que decorrem de cenários informacionais passados em que as tecnologias utilizadas na construção de catálogos apresentavam limitações hoje não mais existentes ou já amenizadas. Espera-se que esta pesquisa possa auxiliar nessa compreensão. 


\section{Referências}

ASSUMPÇÃO, F. S.; SANTOS, P. V. A. da C. A utilização do Resource Description and Access (RDA) na criação de registros de autoridade para pessoas, famílias e entidades coletivas. Encontros Bibli: revista eletrônica de Biblioteconomia e Ciência da Informação, Florianópolis, v. 18, n. 37, p. 203-226, 2013. Disponível em: <http://dx.doi.org/10.5007/15182924.2013v18n37p203>. Acesso em: 30 mar. 2016

AVRAM, H. D. The MARC Pilot Project: Final Report on a Project Sponsored by The Council on Library Resources, Inc. Washington, D.C.: Library of Congress, 1968. Disponível em: <http://www.eric.ed.gov/ERICWebPortal/detail?accno=ED029663>. Acesso em: 30 mar. 2016.

BRANDT, S. Authority Files for Microcomputer Databases. Special Libraries, v. 79, n. 4, p. 296-301, Fall 1988.

BURGER, R. H. Authority Work: the Creation, Use, Maintenance, and Evaluation of Authority Records and Files. Littleton: Libraries Unlimited, 1985.

BURT, P.; KINNUCAN, M. Information Models and Modeling Techniques for Information Systems. Annual Review of Information Science and Technology, p. 175-208, 1990

CARPENTER, M. Main Entry. In: SVENONIUS, E. (Org.). The Conceptual Foundations of Descriptive Cataloging. San Diego: Academic Press, 1989. p. 73-95.

CHAN, L. M. Cataloging and Classification: an Introduction. 3rd ed. Lanham: Scarecrow Press, 2007.

CLACK, D. H. Authority Control and Linked Bibliographic Databases. Cataloging \& Classification Quarterly, v. 8, n. 3/4, p. 35-46, 1988.

COYLE, K. FRBR Before and After: a Look at Our Bibliographic Models. Chicago: American Library Association, 2016. Disponível em: <http://kcoyle.net/beforeAndAfter/>. Acesso em: 30 mar. 2016.

FRBR REVIEW GROUP. World-wide review of the FRBR-Library Reference Model, a consolidation of the FRBR, FRAD and FRSAD conceptual models. [S.I.]: IFLA, 2016. Disponível em: <http://www.ifla.org/node/10280>. Acesso em: 30 mar. 2016.

FUSCO, E. Aplicação dos FRBR na modelagem de catálogos bibliográficos digitais. São Paulo: Cultura Acadêmica, 2011. Disponível em: <http://hdl.handle.net/11449/109186>. Acesso em: 30 mar. 2016.

HITZLER, P.; KRÖTZSCH, M.; RUDOLPH, S. Foundations of Semantic Web technologies. Boca Raton: CRC Press, 2010.

IFLA STUDY GROUP ON THE FRBR. Functional Requirements for Bibliographic Records: Final Report. München: IFLA Section on Cataloguing, 1998. Disponível em: <http://www.ifla.org/files/assets/cataloguing/frbr/frbr.pdf>. Acesso em: $30 \mathrm{mar}$. 2016.

IFLA WORKING GROUP ON FRANAR. Functional Requirements for Authority Data: a Conceptual Model. Den Haag: IFLA, 2013. Disponível em: <http://www. ifla.org/files/assets/cataloguing/frad/frad 2013.pdf>. Acesso em: 30 mar. 2016.

IFLA WORKING GROUP ON FRASAR. Functional Requirements for Subject Authority Data (FRSAD): A Conceptual Model. Den Haag: IFLA, 2010. Disponível em: <http://www.ifla.org/files/assets/classification-and-indexing/functionalrequirements-for-subject-authority-data/frsad-final-report.pdf>. Acesso em: 30 mar. 2016.

MALINCONICO, M. S. The Role of a Machine Based Authority File in an Automated Bibliographic System. In: CARPENTER, M.; SVENONIUS, E. (Eds.). Foundations of Cataloging: a Sourcebook. Littleton: Libraries Unlimited, 1985. Originalmente publicado em: Automation in Libraries: Papers Presented at the CACUL Workshop on Library Automation, Winnipeg, June 22 23, 1974. Ottawa: Canadian Library Association, 1975.

MARC DEVELOPMENT OFFICE. Authorities, a MARC Format: Specifications for Magnetic Tapes Containing Authority Records. Preliminar edition. Washington, D.C.: Library of Congress, 1976. Disponível em: $<$ http://hdl.handle.net/2027/uiug.30112018626959>. Acesso em: 30 mar. 2016.

MAXWELL, R. L. Maxwell's Guide to Authority Work. Chicago: American Library Association, 2002.

MCCALLUM, S. H. MARC: Keystone for Library Automation. IEEE Annals of the History of Computing, v. 24, n. 2, p. 34-49, Apr./Jun. 2002. Disponível em: <http://dx.doi.org/10.1109/MAHC.2002.1010068>. Acesso em: 30 mar. 2016.

RAYWARD, W. B. A History of Computer Applications in Libraries: Prolegomena. IEEE Annals of the History of Computing, v. 24, n. 2, p. 4-15, Apr./Jun. 2002. Disponível em: <http://dx.doi.org/10.1109/MAHC.2002.1010066>. Acesso em: 30 mar. 2016.

SANTOS, P. L. V. A. da C.; PEREIRA, A. M. Catalogação: breve histórico e contemporaneidade. Niterói: Intertexto, 2014.

SAYÃO, L. F. Modelos teóricos em ciência da informação: abstração e método científico. Ciência da Informação, v. 30, n. 1, jan./abr. 2001. Disponível em: <http://dx.doi.org/10.1590/S0100-19652001000100010>. Acesso em: 30 mar. 2016. 
SVENONIUS, E. The Intellectual Foundation of Information Organization. Cambridge: MIT Press, 2000.

TAYLOR, A. G. Introduction to Cataloging and Classification. 10th ed. Westport: Libraries Unlimited, 2006.

TAYLOR, A. G.; JOUDREY, D. N. The Organization of Information. 3rd ed. Westport: Libraries Unlimited, 2009.

TILLETT, B. B. The History of Linking Devices. Library Resources and Technical Services, v. 36, n. 1, p. 23-36, Jan. 1992.

WILLER, M.; DUNSIRE, G. Bibliographic Information Organization in the Semantic Web. Oxford: Chandos Publishing, 2013.

\section{Dados dos autores}

\section{Fabrício Silva Assumpção}

Bacharel em Biblioteconomia pela Universidade Estadual Paulista "Júlio de Mesquita Filho" (UNESP), Campus de Marília (2011), mestre em Ciência da Informação pela UNESP (2013) e doutorando em Ciência da Informação pela UNESP (2014-).

\section{assumpcao.f@gmail.com}

\section{Plácida Leopoldina Ventura Amorim da Costa Santos}

Professora na Universidade Estadual Paulista "Júlio de Mesquita Filho" (UNESP), Campus de Marília. Programa de Pós-Graduação em Ciência da Informação.

\section{placidasantos@gmail.com}

\section{Zaira Regina Zafalon}

Professora na Universidade Federal de São Carlos (UFSCar). Programa de Pós-Graduação em Ciência da Informação (UFSCar).

zaira@ufscar.br

Recebido - Received: 2016-07-25

Aceitado - Accepted: 2016-11-17

\footnotetext{
1 Pesquisa realizada com financiamento da Coordenação de Aperfeiçoamento de Pessoal de Nível Superior (CAPES).

2 São coletivamente denominados Formatos MARC 21: Formato MARC 21 para Dados Bibliográficos, Formato MARC 21 para Dados de Autoridade, Formato MARC 21 para Dados de Itens, Formato MARC 21 para Dados de Classificação e Formato MARC 21 para Informação Comunitária. Disponível em: <https://www.loc.gov/marc/>. Acesso em: 25 fev. 2016.
}

\section{$((c))$ EY}

This work is licensed under a Creative Commons Attribution 4.0

United States License.

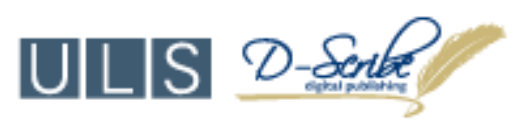

This journal is published by the University Library System of the University of Pittsburgh as part of its

D-Scribe Digital Publishing Program and is cosponsored by the University of Pittsburgh Press. 